rev.relac.int.estrateg.segur.5(2):235-249,2010

\title{
POLÍTICA PREVENTIVA FRENTE A LA TRATA DE PERSONAS SEGURIDAD VERSUS DERECHOS HUMANOS
}

\begin{abstract}
Alejandra Ripoll De Castro*
\section{RESUMEN}

La esclavitud del siglo XXI o trata de personas es un delito que preocupa a los estamentos públicos y privados, por el incremento que ha experimentado en la última década. Entonces, para combatirlo se han puesto en marcha varias estrategias como la protección a las víctimas, la judialización de los tratantes y la prevención, entre otras. Sin embargo, esta última es la más difícil porque requiere la identificación de las causas particulares de vulnerabilidad de las poblaciones en estado de riesgo. Este ensayo revisará los avances en la materia, que es una tarea indispensable para diseñar una verdadera política preventiva que a la postre tenga la finalidad de proteger los Derechos Humanos.

Palabras clave: Trata de personas, política preventiva, vulnerabilidad y derechos humanos.

\section{ABSTRACT}

Slavery on the 21st century or human trafficking is a crime that worries government and civil society, for the increment

\footnotetext{
* Magíster en Relaciones Internacionales, docente-investigadora de tiempo completo, directora de posgrados y editora de la revista de la Facultad de Relaciones Internacionales, Estrategia y Seguridad.
} alejandra.ripoll@unimilitar.edu.co.
\end{abstract}


that it has experienced in the last decade. Then, to fight it, several strategies have been implemented, such as: protection to the victims, prosecution of the traffickers and prevention, among others. Nevertheless, the last one is the most difficult to control because it requires the identification of the particular vulnerability reasons for the population at risk. This essay will review the advances in this matter, which is a necessary task in order to design a true preventive policy that in the end will have the purpose of protecting Human Rights.

Key words: Human Trafficking, preventive policy, vulnerability and human rights

\section{INTRODUCCIÓN}

La Trata de Personas es una realidad, es un delito que afecta a millones de personas alrededor del mundo. La Oficina de Naciones Unidas contra la Droga y el Delito estima que aproximadamente dos y medio millones de personas son tratadas anualmente; en Colombia el estimativo se encuentra alrededor de ochenta mil personas tratadas por año. El Departamento Administrativo de Seguridad DAS señala que cada día salen del país hacia el exterior entre dos y diez personas víctimas de la trata.

La Trata de Personas es la esclavitud del siglo XXI, aún sin usar cadenas, se amenaza a la persona, se intimida su familia y en muchas ocasiones las condiciones de vulnerabilidad en las que se encuentran las víctimas son aprovechadas para vender falsos ideales y sueños. Esta forma de esclavitud degrada al ser humano a la condición de objeto, el cual «se negocia en cadenas mercantiles, se traslada dentro o fuera del país y luego, en el destino final, es sometido a condiciones de explotación y otros fines ilícitos» ${ }^{1}$.

A pesar de ser considerado el tercer delito más lucrativo del mundo, después de las armas y la droga, las cifras siguen siendo una aproximación a la realidad, pues las denuncias son escasas, porque las redes imparten miedo, aterrorizan a sus víctimas y reina la impunidad, con una alta capacidad de intimidación.

Es por esto que un problema de esta magnitud debe afrontarse no solo desde el ámbito de la política criminal o de las normas del derecho administrativo, sino también mediante la construcción y concepción de políticas públicas serias, que den prelación a la política social.

Colombia es considerada por las Naciones Unidas como el tercer país con mayor número de víctimas de trata de personas en el mundo. Es así que, la trata de personas nos sitúa frente a un modelo social diferente en los inicios del siglo XXI. Por lo tanto, es una situación que afecta nuestras sociedades, genera corrupción y nos aqueja como seres humanos.

1 FUNDACIÓN ESPERANZA, SUEÑOS. 2008

POLÍTICA PREVENTIVA FRENTE A LA TRATA DE PERSONAS SEGURIDAD VERSUS DERECHOS HUMANOS 
A pesar de los esfuerzos hechos, tanto por el Estado, como por la sociedad civil, muchos colombianos continúan padeciendo silenciosamente este delito. La mayoría de las víctimas provienen generalmente de ciudades como Barranquilla, Cartagena, Medellín, Bogotá, Manizales, Armenia, Pereira y Cali². Y los principales países de destino de las víctimas colombianas son: Japón, España, Ecuador y Holanda. Adicionalmente, se cree que países como Alemania, Italia, Estados Unidos, Ecuador y Panamá, entre otros, son otros lugares de destino. Así mismo, las autoridades colombianas afirman que entre 20 y 50 personas pasan por Colombia, como punto de tránsito a otros países.

De acuerdo con cifras suministradas por la Fundación Esperanza, en 2009 se registraron en España 865 casos de mujeres víctimas de Trata, de las cuales 568 procedían de países de América Central y del Sur, y de estas 410 víctimas eran colombianas. Estas cifras revelan que la trata es un grave problema que afecta a un gran número de colombianas y colombianos.

Este ensayo se propone revisar los avances en materia de prevención de trata de personas. Para lograr este objetivo, se dividirá en dos partes. Primero, identificar algunas causas de vulnerabilidad para trazar asertivamente una política preventiva. Segundo, revisar el papel de los actores estatales y no estatales comprometidos con el combate de la trata de personas. En ambos casos podemos descubrir que la vulnerabilidad en sí reviste de una complejidad tal, porque involucra aspectos que van desde las pocas oportunidades que brinda el Estado, a parte de la población de insertarse en la dinámica económica que le proporcione un mediano estado de bienestar, como el desplazamiento forzado, la penetración de las bandas criminales transnacionales, entre otras, que han impedido que los esfuerzos de los actores tanto estatales como no estatales tengan un impacto mayor en la prevención.

\section{VULNERABILIDAD Y POLÍTICA PREVENTIVA}

La complejidad del delito de trata de personas es tal que particularizar en algunos de sus aspectos requiere hacer referencia a otros. En el caso de la política preventiva, hay que mirar qué convierte en vulnerables a las personas en determinado espacio geográfico, para el planteamiento asertivo de esa política. En ese sentido, se definirá vulnerabilidad como la incapacidad de resistencia frente a una amenaza. En esa misma dirección, citaremos el paper de Michèle A. Clark, Vulnerabilidad, Prevención y Trata de Personas ${ }^{3}$. En el documento el autor enfatiza en la necesidad de un nuevo paradigma, en el que haya consenso internacional con

2 Cifras del Ministerio del Interior y de Justicia indican que anualmente 35 mil mujeres salen del país, víctimas de este delito, de las cuales el 55\% proviene de veredas, el 35\% de pueblos de tamaño medio y el 10\% de centros urbanos.

3 Revisar el documento de presentación Human Trafficking In North America, como insumo de: La Ley para Prevenir y Sancionar la Trata de Personas en México. Febrero 2009 
relación a un concepto básico como el de víctima, esencial en cualquier esfuerzo por prevenir la trata de personas y proteger los Derechos Humanos de aquellas más vulnerables. En uno de sus argumentos da cuenta de la creencia generalizada de que son víctimas únicamente los tratados con fines de explotación sexual, pero no los tratados con fines de explotación laboral. A la sazón, la falta de acuerdo a la hora de definir víctima dificulta los esfuerzos preventivos que se pueden realizar. Otro de los argumentos que plantea como dificultad en la tarea preventiva es la veracidad de las cifras que obstaculiza diseñar unos parámetros de medición del delito de trata de personas. Lo anterior se debe en parte a la ausencia de consenso, tanto en algunos conceptos, como en el problema de consolidar las cifras. Ahora, ipor qué se presentan estos obstáculos? Una razón sencilla es la magnitud del problema que a la postre no presenta especificidades del fenómeno, requisito importante a la hora de configurar alguna política de prevención.

La política preventiva es tan importante que en el Informe General del Foro de Viena 2008 se hace énfasis en la prevención de la trata, teniendo en cuenta las condiciones de vulnerabilidad y la protección de los Derechos Humanos, porque solo en el momento en que se detecta la vulnerabilidad, es posible generar trabajos preventivos. Así, toda política de prevención que se genere bajo la perspectiva de la vulnerabilidad producirá mejores efectos porque tiene como norte los Derechos Humanos de las víctimas o potenciales víctimas, entonces, el impacto que generará este tipo de políticas es la disminución de los riesgos en el ambiente y evitar el maltrato de los derechos humanos y la dignidad humana.

Es por eso que Judith Dixon, en el documento que aportó en el Foro de Viena 2008, se centra en el impacto que tiene la trata sobre las víctimas; además, analiza la consecuencias físicas, sicológicas, económicas, sociales y políticas de la trata desde la perspectiva humana y ratifica una vez más este fenómeno como una grave violación de los Derechos Humanos. Sin embargo, la contribución principal es la manera como los gobiernos nacionales manejan los Derechos Humanos de las víctimas versus las consecuencias políticas que acarrea la toma de decisión de los entes encargados en esa materia.

En este aparte señala que generalmente los países tienden a incluir la trata de personas dentro de la migración irregular, y desde esta perspectiva generan sus políticas, haciendo énfasis en la legalidad o ilegalidad del tránsito y descuidando las cuestiones humanas, poniendo en riesgo el respeto por los Derechos Humanos; incluso, cuando los países intentan centrar su atención en los tratantes, los tratados corren el riesgo de no ser identificados como víctimas y así ser judicializados en vez de ayudados, aparte de anotar que entre más severas sean las normas migratorias de un país, se posibilitan brutales violaciones a los derechos humanos. Bajo estas condiciones, no hay duda de que la vulnerabilidad de los emigrantes escala a proporciones gigantescas y se convierte en blanco de las bandas de tratantes. Se denota así, que en los diferentes Estados no se avanza mucho en materia de prevención, porque privilegian la seguridad estatal frente a la tutela de los derechos humanos. 
Entonces, la migración, que en la última década se ha acrecentado en forma significativa, agravada por el fenómeno de la globalización, aumenta la vulnerabilidad. Igualmente, en esta sociedad globalizada se presenta una paradoja para los emigrantes, «al mismo tiempo que se da la libre circulación de capitales, ellos viven la creciente restricción de la circulación de personas» ${ }^{4}$, lo que demanda los servicios de los traficantes por los excesivos controles para migrar legalmente y que viabilizan, aún más, la trata de personas. A su vez, esto facilita y aumenta la inseguridad de los emigrantes exponiéndolos a la dominación y abuso de las organizaciones criminales. En este sentido, las políticas migratorias deben ser diseñadas de manera tal que garanticen el debido trato a los migrantes y no se conviertan en políticas sancionadoras para las víctimas del delito de trata de personas al crear diferencias que categorizan a los seres humanos como de primera y segunda clase, lo que incide para que surjan y aumenten nuevas formas de exclusión social.

Entonces, identificar las causas de vulnerabilidad es un ejercicio previo e indispensable para diseñar una verdadera política preventiva. En el caso colombiano, se tendrán en cuenta las expuestas por La Organización Internacional de Migraciones OIM, que asevera que hay ciertas zonas de Colombia donde la población es más propensa o vulnerable a las redes de tratantes, por los siguientes factores: Factores económicos: Falta de empleo, falta de alternativas laborales y pobreza. Factores sociales: Madre soltera, mujeres cabezas de hogar, violencia intrafamiliar, violencia sociopolítica y desastres naturales. Factores culturales: Bajo nivel educativo, preferencia de objetivos económicos, valores sociales distorsionados y desconocimiento de los reales alcances de la trata. Factores psicológicos: Baja autoestima, antecedentes de maltratos, antecedentes de abuso sexual, antecedentes de prostitución y antecedentes delincuenciales.

Es así como las redes de tratantes, aprovechándose de las condiciones de pobreza, del conflicto armado interno y de las ansias de un futuro mejor de muchos colombianos y colombianas, los someten a explotación sexual, a trabajos forzados y a otras situaciones, como en la época de la esclavitud.

Luego hay que tener en cuenta que «en aquellos países donde el Estado logra proveer plenos servicios de seguridad y justicia para todos, se da por sentada la vigencia de los derechos humanos básicos o de «primera generación» ${ }^{5}$ (derechos civiles y políticos). Esta no es, por supuesto, la situación de Colombia; en primer lugar, por no tener una cultura de derechos humanos y, en segundo lugar, por las condiciones precarias de muchos sectores de la población, que se ven abocados a prácticas malsanas o a ejercer oficios poco éticos para sobrevivir. Como señala Consuelo Ahumada, «la reducción del papel social del Estado [...] han empeorado las condiciones

4 CHIAROTTI, Susana. La Trata de Mujeres: Sus Conexiones y Desconexiones con la Migración y los Derechos Humanos. Naciones Unidas-CEPAL. Serie Población y Desarrollo. \# 39. mayo, 2003. p. 20

5 PROGRAMA DE LAS NACIONES UNIDAS PARA EL DESARROLLO. Informe Nacional de Desarrollo Humano Colombia - 2003. Cap.8. p.1. 
de vida de la gran mayoría de la población, que no cuenta con los medios para involucrarse en la «racionalidad del mercado» ${ }^{6}$.

El resultado es una difícil situación socioeconómica de muchos pobladores que frente al desespero buscan formas aparentemente fáciles de hacer dinero, lo que en la mayoría de los casos los convierten en víctimas de las bandas criminales organizadas que los someten a cualquier tipo de labores bajo engaños o a la fuerza. Como bien lo dice Mauricio Rubio, «las mafias logran el control sobre un territorio o un mercado con el uso sistemático de la fuerza» ${ }^{7}$.

En consecuencia, este delito tiene mayor ocurrencia en países en vía de desarrollo o subdesarrollados, y Colombia es uno de los países más golpeados, debido a la problemática local como: altas tasas de desempleo, bajos niveles educativos, conflicto armado, ineficiencia del sistema judicial y una economía débil que difícilmente puede involucrarse en la dinámica del Nuevo Orden Mundial para sacar adelante su población.

Estas razones hacen vulnerable a la población y se vuelven presas fáciles de los tratantes. La inestabilidad política y económica aumenta las posibilidades de convertir un país en materia prima para la trata de personas. Por lo tanto, facilita la labor de los tratantes, que mediante engaño prometen un futuro mejor fuera de las fronteras de las víctimas, ofreciendo mejores salarios, mejores condiciones laborales y mejor calidad de vida. Las condiciones precarias de vida desestabilizan y desplazan a grupos importantes, ante la perspectiva de una vida mejor.

A la sazón, resulta determinante en el combate del delito de la trata de personas la disposición que muestre el Estado para alcanzar logros significativos en política preventiva, porque la voluntad por sí sola es insuficiente. Así, el factor decisivo por la magnitud, complejidad y alcance del delito sigue siendo el trabajo conjunto tanto del sector público como del privado. En este último grupo, la participación de la sociedad civil es definitiva para el logro de las metas propuestas con miras a derrotar o por lo menos a disminuir el impacto de esta agresión de gran magnitud y de consecuencias catastróficas para las personas víctimas de las bandas de tratantes. Sin embargo, para conseguir involucrar a los gobiernos y a la sociedad civil debe haber una gran sensibilización del problema, que se traduzca en poder conocerlo y reconocerlo, para así hacer recomendaciones y prescribir acciones encaminadas a minimizar los efectos drásticos del delito y apuntar a la supresión de este flagelo.

Dentro de la política preventiva nacional, se debe tener en cuenta el desplazamiento forzado por ser esta población altamente vulnerable; generalmente la migración de los desplazados

6 AHUMADA, Consuelo. El Modelo Neoliberal y su impacto en la sociedad colombiana. El Áncora Editores. Bogotá. 1998. p. 246.

7 RUBIO, Mauricio. Crimen e impunidad. Tercer Mundo, Bogotá, 1999. p. 201. 
viene del sector rural al urbano, y la población receptora no tiene infraestructura suficiente para albergar cómodamente a los recién llegados, lo cual conlleva a que se aumenten los índices de pobreza, inseguridad y otros factores negativos en las principales ciudades del país. Además, «no existe una política estatal que tenga en consideración la asistencia a la población receptora, generando conflictos con el grupo recién llegado y agravando los problemas socioeconómicos propios de la zona $\rangle^{8}$. Es de advertir que Colombia es el tercer país del mundo con el mayor número de desplazados, después de Sudán y Angola. Estas duras condiciones terminan en una experiencia bastante traumática para ellos, no solo por las condiciones económicas precarias, sino por otras consecuencias como el desarraigo familiar, cultural y social y las pocas oportunidades de conseguir empleo. Por todo ese ambiente difícil, los desplazados se convierten en un mercado atractivo para las bandas de tratantes.

Por tanto, se requiere trabajo conjunto entre el Estado y la sociedad civil en la búsqueda de soluciones asertivas para la prevención de la trata en esta población. Empero, las instituciones estatales deben ser las principales garantes de los derechos de los desplazados. Así, es imperiosa una acción concertada basada en la confianza mutua, donde se adopten programas coordinados entre los diferentes organismos públicos y privados, así como de las diferentes ramas del poder público. La solidaridad y el compromiso de la sociedad civil son necesarios para que el Estado garantice la seguridad e imparta justicia. En consecuencia, se debe avanzar hacia el establecimiento de mecanismos que disminuyan el grado de vulnerabilidad a la población desplazada con programas de atención inmediata, que no generen situaciones gravosas en el área y logren el retorno lo antes posible.

Para concluir, Colombia, como se ha indicado, es uno de los países más afectados por la práctica de este delito, por los factores determinados anteriormente, no solo para comercio sexual, sino para el tráfico de menores. A pesar de ello, es considerada como uno de los países más avanzados en el combate de ese delito, por cumplir los estándares determinados en la lucha para erradicar la trata de personas.

A la postre, se convierte en prioritario para el estado colombiano adoptar medidas de fondo socioeconómico para enfrentar los problemas de pobreza, educación y desarrollo, a fin de prevenir y combatir el tráfico ilícito de migrantes colombianos y la trata de personas. Por lo tanto, esa es una tarea fundamental para el Gobierno nacional, a fin de disminuir el número de víctimas.

Otra tarea de vital importancia en la erradicación del delito de trata en el contexto colombiano es ajustar el sistema de justicia. En la mayoría de los casos los tratantes no son descubiertos, lo que dificulta el sometimiento de estos a la ley. Una de las causas es que en la mayoría de los

8 FUNDACIÓN ESPERANZA, Op. cit., p. 18. 
tratados internacionales y propuestas de la sociedad civil se centran más en la preocupación de las víctimas, con poco énfasis en el castigo de los tratantes.

De ahí que «un excesivo énfasis en la víctima puede dejar de lado lo que debe ser la preocupación fundamental de la justicia, que es la investigación y sanción de los promotores y gestores del crimen, en este caso, los traficantes» ${ }^{9}$. Además de ser «una salida fácil y cómoda para evitar la tarea más difícil, comprometida y austera, es decir, el combate a los responsables de estos crímenes» ${ }^{10}$.

Por consiguiente, apuntar a un equilibrio entre persecución y sanción a los traficantes y prevención y protección a las víctimas, es ideal para lograr resultados; es decir, atacar tanto la oferta como la demanda ${ }^{11}$.

Para finalizar esta parte es importante precisar que el universo de víctimas de trata en Colombia es más amplio que el que tiene el imaginario colectivo identificado. Comúnmente se conciben las mujeres y hombres en etapa de adulto-joven, a los niños y niñas de escasos recursos económicos y con altos niveles de analfabetismo. Sin embargo, se han detectado casos de trata en personas de diferente estrato socio-económico y educativo, como los universitarios y profesionales jóvenes que no son considerados como vulnerables por su nivel de educación ${ }^{12}$.

\section{ACTORES}

Las tres grandes preocupaciones de los actores nacionales e internacionales son y han sido el proteger a las víctimas de trata, sancionar a los tratantes y prevenir la ocurrencia de la misma. Sin embargo, en este trabajo la revisión del papel de los diferentes actores hará énfasis en la tarea más difícil que es la prevención. Así, se apelará al institucionalismo liberal, porque sistematiza algunos de los factores que se requieren para prevenirla. Además, constituye algunos requisitos previos e indispensables para la adopción de cualquier medida a favor de minimizar el impacto del delito de la trata de personas, es decir, entender y reflejar el problema existente mostrando elementos que la causan y cómo evitarlo. Por tanto, el contenido de este marco debe tomarse como una introducción para la reflexión sobre la importancia de

9 CHIAROTTI,. Op. cit. p. 21.

10 Ibid., p. 24.

11 EVERTS, Daan. The Brown Journal of World Affairs. Vol. 10. Summer/Fall. 2003. Citado por: Andrea M. Bertone. Transnational Activism to Combat Trafficking in Persons.College Park Scholars International Studies. p. 9. (traducción libre)

12 Revisar: RIPOLL, Alejandra La Existencia de Población Vulnerable a la Trata de Personas dentro de las universidades de Bogotá. 2010. 
prevenir la trata de personas a través de acciones conjuntas entre los gobiernos y demás actores de la sociedad internacional.

Dentro de esta perspectiva, la estructura del sistema colombiano ha propiciado la coordinación de políticas estatales a favor de combatir la trata de personas en el territorio nacional, coordinado con organizaciones internacionales. Estos planes de acción de política exterior han mostrado su capacidad para disminuir el delito en Colombia y estar a la vanguardia para detectar redes de tratantes. Además, ha evidenciado su capacidad de repercutir sobre otros temas como la coordinación de programas sociales esenciales en la lucha contra este flagelo, sobre todo en este país, que pertenece al grupo de países en vía de desarrollo y como consecuencia es un semillero para la trata de personas ${ }^{13}$ por las condiciones socioeconómicas existentes.

Tal como se ha indicado anteriormente, existe una multiplicidad de actores interesados en el combate efectivo de la trata de personas, tales como: los Estados, organizaciones internacionales, organizaciones no estatales y grupos de la sociedad civil, que trabajan activamente en pro de que se respeten los derechos humanos y civiles de las víctimas, por un lado, y por otro, la persecución a los tratantes.

Ahora, la manera de hacer que el trabajo conjunto de los actores repercuta positivamente en la tarea de prevenir y sancionar la trata de personas está en el planteamiento de estrategias comunes e implementación de las mismas. Sin embargo, no todas las veces el resultado es el esperado por unos y por otros, porque cada uno de los actores involucrados en esa labor entiende de forma distinta el fenómeno de la trata; para algunos sectores es un problema de seguridad estatal, mientras que para otros es un problema de derechos humanos.

Además, muchos gobiernos todavía responden a la trata a través de políticas restrictivas, lo que hace más vulnerable a los migrantes frente a los traficantes ${ }^{14}$.

Estos modelos dan prioridad a las leyes y no a los derechos de las personas traficadas. Esto constituye un retraso significativo para el logro del objetivo final, en este caso la prevención; además, que va en detrimento de la protección de los derechos de las víctimas.

Es así que los diversos actores estatales o no estatales deben ajustar su comportamiento con respecto a otros actores mediante un proceso de coordinación de políticas y cooperación, en el

13 RIPOLL, Alejandra. Colombia: Semillero para la Trata de Personas. En: Revista de Relaciones Internacionales, Estrategia y Seguridad. Universidad Militar Nueva Granada. Vol 3. No. 1. Bogotá. 2008.

${ }^{14}$ BERTONE. Andrea M. Transnational Activism to Combat Trafficking in Persons.College Park Scholars International Studies. P. 16 (traducción libre) 
que implique el concurso activo en proyectos puntuales y realizables de tipo económico encaminados a proporcionar un mediano estado de bienestar para que merme la vulnerabilidad.

El compromiso de los diferentes actores es indispensable en la prevención y combate del delito, sobre todo los Estados que deberían impulsar nuevas formas de combatirlo, pero en un escenario común «en el cual los programas y las políticas unilaterales se sustituyan por programas conjuntoss ${ }^{15}$, porque la solución al problema de la trata de personas exige una estrecha colaboración entre los países de origen, de destino y, por ende, de aquellos por los que transitan las víctimas de ese flagelo. Por lo tanto, como convoca la Vicepresidencia de la República de Colombia, hay que «aunar esfuerzos y fortalecer los lazos de cooperación entre Estado y sociedad» ${ }^{16}$, y una acción concertada basada en la confianza mutua en donde se adopten programas coordinados entre los diferentes organismos públicos y privados, así como de las diferentes ramas del poder público en los países involucrados en la problemática. La solidaridad y el compromiso de la sociedad civil son necesarios para que el Estado garantice la seguridad e imparta justicia.

En este sentido, podemos concluir que a pesar de los esfuerzos mancomunados entre Estados, organizaciones internacionales y organizaciones no estatales, no se ha logrado combatir y prevenir eficazmente la trata porque «no hay coordinación global formal de sus esfuerzos y esta falta de coordinación puede estar actualmente exacerbando el problema» ${ }^{17}$.

Pero se presenta otro problema que ayuda a exacerbar la trata y se centra en la definición de amenaza, pues esta debe ser compatible, desde la óptica de los diferentes Estados, con el concepto de seguridad e interés nacional. «Los tipos de amenazas diferencian de manera sustantiva diversas regiones del mundo y subregiones dentro de ella» ${ }^{18}$, lo que dificulta el alcance de una meta común. Así, se requiere con urgencia la incorporación de variables que permitan visualizar los problemas desde una perspectiva global y no regional o subregional. Esto involucra directamente la definición de la agenda de los Estados del mundo que padezcan la latente amenaza del crimen organizado internacional en materia de narcotráfico, terrorismo y la trata de personas, entre otros.

La manera de lograrlo es a través de la cooperación internacional. Sin embargo, en problemas como la trata de personas no ha sido suficiente en la actualidad, como bien lo planteó Ana María Díaz, «preocupa que hoy, ante la vigencia de un sistema universal de protección a los Derechos

15 TORO Celia. Narcotráfico: lo que la interdependencia no nos explicó. La política Exterior de México: enfoques para su análisis, El Colegio de México, Instituto Matías Romero de Estudios Diplomáticos, México. 1997. p. 74.

16 Ibid., p. 8.

17 BERTONE. Op. cit., p. 15 (traducción libre).

18 ROJAS A. Francisco. Cooperación y Seguridad Internacional en las Américas: nuevos conceptos, nuevos riesgos, nuevas amenazas. En: Cooperación y seguridad Internacional en las Américas. FLACSO-Chile. Ed. Nueva Sociedad. Caracas- Venezuela. 1999. p. 24 
Humanos, la trata de personas no solamente persista, sino que se aumente de una forma alarmante teniendo como base el ejercicio abusivo de poder sobre las personas en condiciones más vulnerables de la sociedad $\gg^{19}$.

Por eso, se requiere de un cambio de actitud responsable por parte de los hacedores de política con la adhesión de todas las esferas sociales para producir efectos certeros en el combate de la trata de personas. Gallicchio lo expresa así: «Los cambios necesarios no pueden ser abordados por organizaciones aisladas ni por proyectos puntuales, sino que deberán establecerse fórmulas sistemáticas y persistentes de interacción social $\gg^{20}$. Estos cambios metódicos que sugiere Gallicchio lograrán crear conciencia de la existencia real del problema, además de enfrentarlo sin avergonzarse y muchos menos ocultarlo como ocurre en muchos países explotados y explotadores.

Colombia cuenta con varias instituciones nacionales e internacionales dedicadas al combate de la trata de personas, con unas tareas específicas, entre ellas: la Fiscalía General de la Nación, que es la institución encargada de dirigir las investigaciones relacionadas con los delitos de trata. En los casos de trata externa, los consulados de Colombia en el exterior reciben las denuncias, luego las remiten al Ministerio de Relaciones Exteriores para que, a su vez, las envíe a la Dirección de Asuntos Internacionales de la Fiscalía General de la Nación. En el caso de menores de edad, las denuncias pasan a conocimiento del Instituto Colombiano de Bienestar Familiar ICBF. El Ministerio de Relaciones Exteriores, que suministra información a los funcionarios consulares, trabaja con los cónsules extranjeros acreditados en Colombia para identificar potenciales víctimas, asiste a las víctimas en el exterior y remite los casos de trata a la Fiscalía General de la Nación. El Grupo Humanitas, de la Policía, cumple con funciones de verificación, análisis de información y adelanta investigaciones relacionadas con la trata de personas; de igual forma, coordina acciones interinstitucionales y de prevención. El DAS/Interpol lleva a cabo operativos de rescate con apoyo de autoridades extranjeras; participa en labores de investigación y realiza acciones para judicializar a los tratantes. La Procuraduría General de la Nación, con relación a la Trata, cuenta con la Procuraduría Delegada para la Defensa del Menor y la Familia. La Oficina de las Naciones Unidas Contra la Droga y el Delito-UNODC apoya al Estado colombiano en el diseño e implementación de respuestas coordinadas y efectivas a los desafíos de la trata de personas como modalidad de crimen transnacional. La OIM-Colombia ha trabajado desde 2002 en cuatro áreas directamente relacionadas con la trata: fortalecimiento institucional, sensibilización y prevención, investigación y asistencia a víctimas.

19 DÍAZ, Ana María. El tráfico de mujeres, niñas y niños: Un asunto de Derechos Humanos. Tráfico de personas en Colombia. Primer Encuentro Nacional sobre Tráfico de Personas. Memorias Bogotá. Citado por: Fundación Esperanza. Trata de Personas y Desplazamiento Forzado. Estudio exploratorio sobre la vulnerabilidad de la trata de personas en poblaciones en situación de desplazamiento en Aguablanca, Cali-Colombia. Memo O. y Cía. LTDA. Cali - Colombia. 2004. p. 40

${ }^{20}$ GALLICCHIO, Enrique. El desarrollo local en América Latina, Estrategia Política basada en la Construcción de Capital Social. Córdoba, Argentina. Mayo. 2004. p. 18 
Colombia no solo cuenta con los organismos mencionados, sino que se preocupa por desarrollar nuevos procedimientos y programas; es así que en los últimos años se destaca la creación del Programa de Prevención, Asistencia y Reintegración para las víctimas de la Trata de Personas, cuyo objetivo fundamental ha sido crear instrumentos de asistencia y prevención. También, se crearon dos bases de datos: la primera, denominada «Base de Asistencia», que incluye los casos remitidos a la OIM-Colombia, bien sea para asistir a las víctimas o para darles información. El propósito de dicha base de datos es establecer el perfil de las personas objeto de la trata para brindarle asistencia a través de una institución gubernamental u ONG. La segunda base de datos se construye a partir de la «Línea de Trata de Personas y Migración Informada», que es una línea telefónica habilitada para brindar información, recibir denuncias anónimas y averiguar por la legalidad de ofertas de trabajo, además de buscar desaparecidos y víctimas de trata, entre otros; ha servido como herramienta de contacto directo con las víctimas, para brindar una primera asistencia y remitir casos a las autoridades.

Después de esta revisión de los actores involucrados se puede concluir que Colombia está seriamente comprometida y hace significativos esfuerzos para combatir y prevenir la trata de personas. Esto se puede afirmar con base en el Informe Trafficking Victims Protection Act-Minimum Standards for the Elimination of Trafficking in Persons (TVPAs), que clasifica a Colombia dentro del grupo uno (1); no obstante, todos estos actores deberán seguir trabajando con mano firme y dedicación para disminuir no solo la vulnerabilidad sino para eliminar sus causas y así cumplir el objetivo principal: la prevención de la esclavitud del siglo XXI. Con todo, para avanzar en la política preventiva es necesario identificar los factores de riesgo para hacer las debidas recomendaciones con miras a transformar el medio grupal y el individual. Pero, «esta transformación solo es posible a través del concurso y corresponsabilidad de entidades gubernamentales y organizaciones de la sociedad civil, la comunidad y los individuos ${ }^{21}$.

\section{CONCLUSIONES}

La trata de personas es una violación a los derechos humanos. En los últimos años, frente al aumento del delito de trata de personas en el panorama mundial, la sociedad internacional ha demostrado una creciente capacidad de respuesta. Esta se ha desarrollado no solo a nivel internacional, sino a nivel interno. Internamente, se trasladan las victimas de zonas de pobreza a zonas donde se puedan explotar económicamente por diversos medios. A nivel internacional se da en un esquema regional, alrededor de sus fronteras y más allá de ellas.

21 FUNDACIÓN ESPERANZA. Trata de Personas y Desplazamiento Forzado. Estudio exploratorio sobre la vulnerabilidad de la trata de personas en poblaciones en situación de desplazamiento en Aguablanca, CaliColombia. Memo O. y Cia LTDA. Cali - Colombia. 2004. p. 158. 
Colombia, es un país subdesarrollado que padece los embates de este delito. Se calcula que más o menos 80.000 colombianos y colombianas son tratados anualmente. Las causas de vulnerabilidad son variadas como: factores económicos, factores sociales, factores culturales y factores psicológicos.

Estas razones hacen vulnerable a la población y se vuelven presas fáciles de los tratantes. La inestabilidad política y económica aumenta las posibilidades de convertir un país en materia prima para la trata de personas. Por tanto, facilita la labor de los tratantes, que mediante engaño prometen un futuro mejor fuera de las fronteras de las víctimas, ofreciendo mejores salarios, mejores condiciones laborales y mejor calidad de vida. Las condiciones precarias de vida desestabilizan y desplazan a grupos importantes, ante la perspectiva de una vida mejor.

Frente a esta situación, la solución de muchos colombianos y colombianas es involucrarse en procesos migratorios, muchas veces sin el lleno de las formalidades legales, lo que convierte a muchos emigrantes en botín para los tratantes.

En respuesta, Colombia ha trazado políticas de prevención de la trata de personas, pero la delincuencia organizada ha penetrado casi en todo el mundo, haciendo muy difícil la tarea; sin embargo, este país es considerado como uno de los más avanzados en el combate de ese delito, por cumplir los estándares determinados en la lucha para erradicarlo.

Bajo esta premisa se convierte en prioritario para el Estado colombiano, en la política preventiva, adoptar medidas de fondo socioeconómico para enfrentar los problemas de pobreza, educación y desarrollo, a fin de prevenir y combatir, no solo la trata de personas, sino el tráfico ilícito de emigrantes colombianos. Además, ajustar el sistema de justicia.

El esquema de esta tarea debe estar enmarcado por principios de corresponsabilidad y reciprocidad para lograr con una estrategia común la aplicación de mecanismos eficientes que lleve a resultados positivos. Esto se logrará con una acción consensuada entre gobierno y sociedad civil donde se privilegien los derechos humanos. 


\section{BIBLIOGRAFÍA}

- AHUMADA, Consuelo. El Modelo Neoliberal y su impacto en la sociedad colombiana. El Áncora Editores. Bogotá. 1998

- BeRTONe. Andrea M. Transnational Activism to Combat Trafficking in Persons.College Park Scholars International Studies.

- CHIAROTTI, Susana. La Trata de Mujeres: Sus Conexiones y Desconexiones con la Migración y los Derechos Humanos. Naciones Unidas-CEPAL. Serie Población y Desarrollo. \# 39, mayo, 2003. p. 20

- CLARK, Michèle A. Vulnerabilidad, Prevención y Trata de Persona. Foro de Viena para Combatir la Trata de Personas, 13-15 de febrero de 2008 - Se busca pasaporte: combate contra la trata de personas y el trabajo forzoso

- DíAZ, Ana María. El tráfico de Mujeres, niñas y niños: Un asunto de Derechos Humanos. Tráfico de personas en Colombia. Primer Encuentro Nacional sobre Tráfico de Personas. Memorias Bogotá. Citado por: Fundación Esperanza. Trata de Personas y Desplazamiento Forzado. Estudio exploratorio sobre la vulnerabilidad de la trata de personas en poblaciones en situación de desplazamiento en Aguablanca, Cali-Colombia. Memo O. y Cia LTDA. Cali - Colombia. 2004. p. 40

- DIXON, Judith. Foro de Viena para Combatir la Trata de Personas, 13-15 de febrero de 2008 - Se busca pasaporte.

- $\quad$ EVERTS, Daan. The Brown Journal of World Affairs. Vol. 10. Summer/Fall. 2003. Citado por: Andrea M. Bertone. Transnational Activism to Combat Trafficking in Persons.College Park Scholars International Studies.

- FUNDACIÓN ESPERANZA, Tráfico de Personas-Naufragio de Sueños. Bogotá 2003.

- _ _ Trata de Personas y Desplazamiento Forzado. Estudio exploratorio sobre la vulnerabilidad de la trata de personas en poblaciones en situación de desplazamiento en Aguablanca, Cali-Colombia. Memo O. y Cía. LTDA. Cali - Colombia. 2004. p. 158.

- GALLICCHIO, Enrique. El desarrollo local en América Latina, Estrategia Política basada en la Construcción de Capital Social. Córdoba, Argentina. Mayo, 2004.

- $\quad$ HUMAN TRAFFICKING IN NORTH AMERICA. Documento de presentación como insumo de: La Ley para Prevenir y Sancionar la Trata de Personas en México. Febrero 2009

- MINISTERIO DEL INTERIOR Y DE JUSTICIA, República de Colombia, Trata de Personas. Ley 985 de 2005.

- $\quad$ PROGRAMA DE LAS NACIONES UNIDAS PARA EL DESARROLLO. Informe Nacional de Desarrollo Humano Colombia - 2003. Cap. 8.

- $\quad$ RIPOLL, Alejandra. Colombia: Semillero para la Trata de Personas. En: Revista de Relaciones Internacionales, Estrategia y Seguridad. Universidad Militar Nueva Granada. Vol 3, No. 1. Bogotá. 2008. 
- _ _ _ La Existencia de Población Vulnerable a la Trata de Personas dentro de las universidades de Bogotá. 2010 .

- ROJAS A. Francisco. Cooperación y seguridad Internacional en las Américas: nuevos conceptos, nuevos riesgos, nuevas amenazas. En: Cooperación y seguridad Internacional en las Américas. FLACSO-Chile. Ed. Nueva Sociedad. Caracas- Venezuela. 1999. p. 24

- $\quad$ RUBIO, Mauricio. Crimen e impunidad. Tercer Mundo, Bogotá, 1999. p. 201.

- TORO Celia. Narcotráfico: lo que la interdependencia no nos explicó. La política Exterior de México: enfoques para su análisis, El Colegio de México, Instituto Matías Romero de Estudios Diplomáticos, México. 1997. 